\title{
anthropozoologica
}

$2020 \cdot 55 \cdot 11$

\section{La classification des animaux sauvages chez un naturaliste et médecin du Xe siècle Aḥmad Ibn Abī al-Aš'at}

Kaouthar LAMOUCHI CHEBBI \& Mehrnaz KATOUZIAN-SAFADI

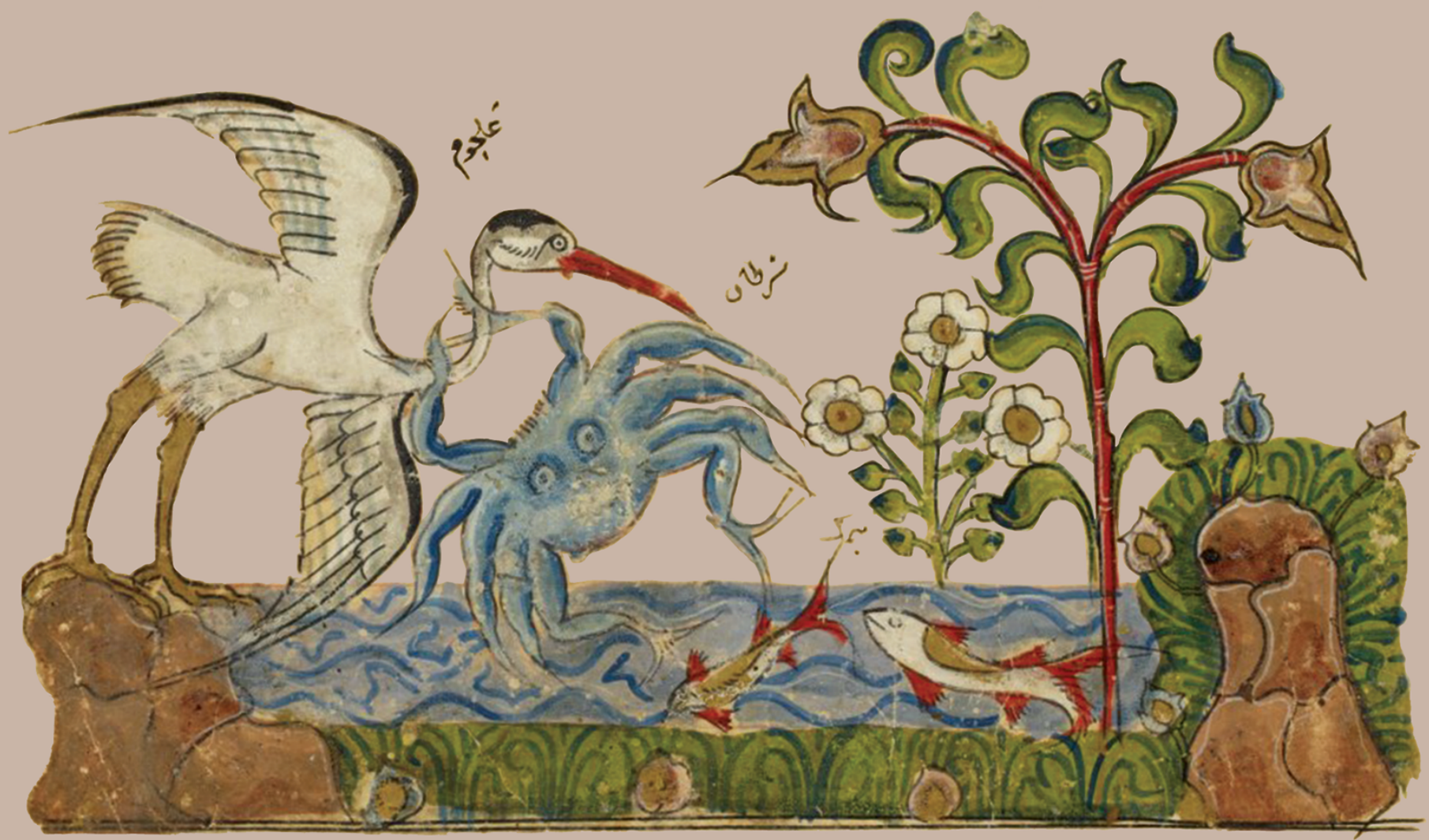


Directeur de la publication / Publication diREctor: Bruno David

Président du Muséum national d'Histoire naturelle

RÉDACTRICE EN CHEF / EDITOR-IN-CHIEF: Joséphine Lesur

RÉDACTRICE / EDITOR: Christine Lefèvre

RESPONSABLE DES ACTUALITÉS SCIENTIFIQUES / RESPONSIBLE FOR SCIENTIFIC NEWS: Rémi Berthon

ASSISTANTE DE RÉDACTION / ASSISTANT EDITOR: Emmanuelle Rocklin (anthropo@mnhn.fr)

MISE EN PAGE / PAGE LAYOUT: Emmanuelle Rocklin, Inist-CNRS

COMITÉ SCIENTIFIQUE / SCIENTIFIC BOARD:

Louis Chaix (Muséum d'Histoire naturelle, Genève, Suisse)

Jean-Pierre Digard (CNRS, Ivry-sur-Seine, France)

Allowen Evin (Muséum national d'Histoire naturelle, Paris, France)

Bernard Faye (Cirad, Montpellier, France)

Carole Ferret (Laboratoire d'Anthropologie Sociale, Paris, France)

Giacomo Giacobini (Università di Torino, Turin, Italie)

Lionel Gourichon (Université de Nice, Nice, France)

Véronique Laroulandie (CNRS, Université de Bordeaux 1, France)

Stavros Lazaris (Orient \& Méditerranée, Collège de France - CNRS - Sorbonne Université, Paris, France)

Nicolas Lescureux (Centre d'Écologie fonctionnelle et évolutive, Montpellier, France)

Marco Masseti (University of Florence, Italy)

Georges Métailié (Muséum national d'Histoire naturelle, Paris, France)

Diego Moreno (Università di Genova, Gènes, Italie)

François Moutou (Boulogne-Billancourt, France)

Marcel Otte (Université de Liège, Liège, Belgique)

Joris Peters (Universität München, Munich, Allemagne)

François Poplin (Muséum national d'Histoire naturelle, Paris, France)

Jean Trinquier (École Normale Supérieure, Paris, France)

Baudouin Van Den Abeele (Université Catholique de Louvain, Louvain, Belgique)

Christophe Vendries (Université de Rennes 2, Rennes, France)

Denis Vialou (Muséum national d'Histoire naturelle, Paris, France)

Jean-Denis Vigne (Muséum national d'Histoire naturelle, Paris, France)

Arnaud Zucker (Université de Nice, Nice, France)

COUVERTURE / COVER:

Cormoran, poissons et crabe (Bibliothèque Nationale de France, Paris: Ms Arabe 3465, fol. 57). Enluminure arabe, Syrie, c. 1200-1220. Illustration de Kalla wa dimna, recueil de fables orientales (tirées du Panchatantra, contes sanskrit) et traduit du Persan Pehlevi vers l'Arabe par Abdallah lbn al-Muqaffa ( $\mathrm{c}$. 756) / Cormorant, fish and crab (Bibliothèque Nationale de France, Paris: Ms Arabe 3465, fol. 57). Arabic illumination, Syria, c. 1200-1220. Illustration from Kalla wa dimna, collection of oriental fables (taken from the Panchatantra, Sanskrit tales) and translated from Persian Pehlevi into Arabic by Abdallah Ibn al-Muqaffa ( $†$ c. 756).

Anthropozoologica est indexé dans / Anthropozoologica is indexed in:

- Social Sciences Citation Index

- Arts \& Humanities Citation Index

- Current Contents - Social \& Behavioral Sciences

- Current Contents - Arts \& Humanities

- Zoological Record

- BIOSIS Previews

- Initial list de l'European Science Foundation (ESF)

- Norwegian Social Science Data Services (NSD)

- Research Bible

Anthropozoologica est distribué en version électronique par / Anthropozoologica is distributed electronically by:

- BioOne ${ }^{\circledR}$ (http://www.bioone.org)

Anthropozoologica est une revue en flux continu publiée par les Publications scientifiques du Muséum, Paris, avec le soutien du CNRS Anthropozoologica is a fast track journal published by the Museum Science Press, Paris, with the support of the CNRS.

Les Publications scientifiques du Muséum publient aussi / The Museum Science Press also publish: Adansonia, Zoosystema, Geodiversitas, European Journal of Taxonomy, Naturae, Cryptogamie sous-sections Algologie, Bryologie, Mycologie, Comptes Rendus Palevol.

Diffusion - Publications scientifiques Muséum national d'Histoire naturelle

CP $41-57$ rue Cuvier F-75231 Paris cedex 05 (France)

Tél. : 33 (0)1 40794805 / Fax: 33 (0)1 40793840

diff.pub@mnhn.fr / http://sciencepress.mnhn.fr

(C) Publications scientifiques du Muséum national d'Histoire naturelle, Paris, 2020

ISSN (imprimé / print): 0761-3032 / ISSN (électronique / electronic): 2107-08817 


\title{
La classification des animaux sauvages chez un naturaliste et médecin du Xe siècle Aḥmad lbn Abī al-Ašat
}

\author{
Kaouthar LAMOUCHI CHEBBI \\ Mehrnaz KATOUZIAN-SAFADI \\ Laboratoire Sciences, Philosophie, Histoire (SPHERE), CNRS, Université Paris 7 Denis Diderot, \\ Bâtiment Condorcet, case 7093, 5 rue Thomas Mann, F-75205 Paris cedex 13 (France) \\ kaouthar.chebbi2@gmail.com \\ katouzian.safadi@orange.fr
}

Soumis le 12 décembre 2019 | Accepté le 1er juin 2020 | Publié le 31 juillet 2020

MOTS CLÉS

Zoologie arabe médiévale, classification, quatre éléments, lbn Abì al-Aš at. animaux sauvages.

KEY WORDS

Medieval Arabic zoology, classification four elements, Ibn Abì al-Aš at, wild animals.
Lamouchi Chebbi K. \& Katouzian-Safadi M. 2020. - La classification des animaux sauvages chez un naturaliste et médecin du Xe siècle Ahmad lbn Abī al-Aš aț, in Brémont A., Boudes Y., Thuault S. \& Ben Saad M. (éds), Appréhender les catégories zoologiques dans les sociétés du passé. Anthropozoologica 55 (11): 159-172. https://doi.org/10.5252/ anthropozoologica2020v55a11. http://anthropozoologica.com/55/11

\section{RÉSUMÉ}

Nous examinons la classification des animaux d'un savant du Xe siècle, Ahmad Ibn Abī al-Aš́at, praticien et théoricien reconnu par ses pairs dans le domaine de la médecine. Cet auteur avait une excellente connaissance des œuvres de ses prédécesseurs grecs, et notamment de Galien. Dans son ouvrage Kitāb al-Hayawān, son regard original aborde le domaine de la classification des animaux sauvages par leur constitution et leur composition par les quatre éléments. Cette classification qui diffère de celle d'Aristote ou du savant arabe al-Ğahiz (IXe siècle), intègre cependant des éléments d'observation de certains savants naturalistes concernant l'environnement. Dans cet article, nous souhaitons approfondir l'analyse d'un chapitre de son œuvre, sur la classification des animaux sauvages, particulièrement remarquable par son aspect théorique et systématique. Ibn Abī al-Aš at propose des lois générales qui permettent de placer chaque animal, connu ou non de l'auteur, dans une catégorie qui lui correspond. Seize catégories sont ainsi définies et illustrées par des exemples d'animaux réels. Nous examinons les relations entre la construction de ces catégories et divers domaines de l'art médical.

\section{ABSTRACT}

Wild animals categorization by the tenth century naturalist doctor Ahmad Ibn abi al-Ašst.

We consider the classification of animals of a scholar of the 10 th century, Aḥmad Ibn Abī al-Aš at, a recognized practitioner and theoretician in the field of medicine. This author had a wide knowledge of the works of his Greek predecessors, including Galen. His original approach addresses the field of wild animal taxonomy (in his book Kitäb al-Hayawān) by their constitution and composition by the four elements. While taking into account other factors, mainly the living environment, this classification differs from that of Aristotle or the Arab scholar al-Ğahiz (9th). In this paper, we delve deeper into the analysis of a chapter of his work, on the classification of wild animals, which is particularly remarkable for its theoretical and systematic aspect. Ibn Abī al-Aš at proposes general laws that allow the reader to place any animal, known or unknown to the author, in a corresponding category. Sixteen categories are thus defined and illustrated by examples of real animals. In this article, we examine the relationships between medical Art and the establishment of these categories. 


\section{INTRODUCTION}

Nous examinons quelques aspects de la classification des animaux d'un auteur appartenant à la civilisation médiévale arabo-islamique. Cette civilisation résulte du syncrétisme des cultures orales et écrites du vaste Proche-Orient en intégrant les diverses religions (juive, chrétienne, islamique), les agnostiques, diverses philosophies et de multiples langues régionales (Gutas 1998), comme la langue syriaque. Par économie de termes, dans la suite de l'article nous emploierons les qualificatifs «Islamique - Arabo-persane», «Islamique - Arabe» ou "Arabe» sans restriction pour une langue ou une religion unique (KatouzianSafadi 2014). Pour les translittérations des caractères arabes en caractères latins se reporter à la Table 1.

Nous nous intéressons à une des œuvres d'Ahmad Ibn Abī al-Ašs at ou IbnAA († 970), le Kitāb al-Haywān ou Kitāb al-H ${ }^{1}$. D'après le bibliographe Ibn Abī Ușaybi a (1203-1270), IbnAA, d'origine persane, aurait fui sa terre natale vers Mossoul, en Irak, pour des raisons financières. Devenu médecin sur le tard, il doit sa célébrité à sa contribution à la guérison inespérée de l'un des fils de Naser al-Dawla, prince hamdanide de Mossoul. IbnAA mourut en laissant beaucoup d'élèves, plusieurs ouvrages médicaux ainsi que des commentaires et des résumés sur des travaux de Galien (Sezgin 1995: 254-257).

Remke Kruk fut la première à réaliser une étude de très nombreux fragments du texte de Kitäb al-H d'IbnAA. Elle s'est basée pour cela sur un manuscrit unique, le MS Oxford, Bodl. Hunt 534/6 (Kruk 2001: 119). Nous avons travaillé à partir de l'édition irakienne de ce même manuscrit, réalisée par Ahmad Harbì à Bagdad (Harbī 2008). Cet ouvrage sur les êtres vivants se divise en trois grands chapitres (maqāla): le premier est consacré aux humains, le deuxième aux animaux domestiques et le troisième aux animaux sauvages ${ }^{2}$; nos analyses portent plus particulièrement sur ce dernier chapitre. Nos recherches s'inscrivent dans la continuité des travaux de $\mathrm{R}$. Kruk et nous poursuivons ainsi ses dernières conclusions sur l'importance des propriétés humorales des animaux (Kruk 2001: 164, lignes 13, 14). Notre but est de mettre en évidence que cette œuvre est imprégnée par les théories et par les pratiques médico-pharmaceutiques de l'auteur. Pour cela, nous comparons la méthode de classification d'IbnAA à celle d'autres naturalistes et zoologistes comme Ğăhị et à celle des médecins et des pharmaciens de son époque.

Dans un premier temps, nous rappelons le cadre théorique de la pensée des naturalistes à l'époque d'IbnAA en relation avec sa classification des animaux sauvages. Nous présenterons ensuite très brièvement quelques types de classifications des animaux du IXe au XIe siècle. Cela nous permettra d'aborder l'œuvre d'IbnAA et de comprendre l'interdépendance des trois chapitres du livre. Nous examinerons enfin le troisième chapitre concernant les animaux sauvages en le situant dans le cadre de la philosophie naturelle, de la médecine et de la pharmacie de cette époque.

1. Le terme "hayy» en arabe signifie "vif, vivant», "hayawān» signifie "être animé», "être vivant» et par extension, il peut désigner l'"animal». Dans la suite de l'article, nous nommerons le livre d'IbnAA, Kitäb $H$.

2. Nous expliciterons plus loin ce terme simplificateur de "sauvage», employé ici.

\section{LE CADRE THÉORIQUE: RELATIONS RÉCIPROQUES ENTRE LES ÉLÉMENTS, LES HUMEURS, LE TEMPÉRAMENT ET LE MILIEU}

Pour aborder la classification d'IbnAA, il importe de rappeler que la théorie humorale des pathologies, systématisée par Galien, constituait les fondements de la pensée philosophique et médicale des savants de l'Antiquité à la période médiévale, mais aussi après la Renaissance. Quelques développements s'imposent donc sur les répercussions de cette théorie dans des domaines comme la pharmacie, l'alchimie, la médecine et la physiognomonie.

Le principe des quatre éléments (le Feu, l'Eau, l'Air, la Terre) et de leurs qualités principales (la Chaleur, la Froideur, l'Humidité et la Sécheresse) se forme progressivement au cours d'une longue histoire qui commence avant le Ve siècle av. J.-C. et se poursuit bien au-delà du XIIIe siècle ap. J.-C. Les centres de cette pensée en formation migrent probablement de l'Égypte (David 2004: 133-151) vers la Grèce puis vers le Proche-Orient et s'étendent dans le vaste empire islamique (Katouzian-Safadi \& Ben Saad 2019: 212-216). À ces éléments et leurs qualités peut également être associé un autre critère, celui de la densité (légèreté ou lourdeur). Nous l'évoquerons dans la section consacrée aux modes de classification.

Hippocrate (c. 460-370 av. J.-C.) et le corpus hippocratique, formé de l'ensemble des textes qui lui sont attribués, forment l'un des points d'ancrage de l'histoire de ces théories. Selon Hippocrate, pour traiter les maladies, il faut connaître la nature de l'homme, sa physis. Une des théories courantes au Ve siècle av. J.-C. était l'existence de deux humeurs, le sang, humeur de la santé, et la bile ou phlegme, humeur de la maladie. Le traité De la Nature de l'homme est l'œuvre de Polybe, le gendre d'Hippocrate. Six siècles plus tard, Galien reprend ce traité comme œuvre d'Hippocrate et déclare que la nature de l'homme est constituée de quatre humeurs: sang, phlegme, bile jaune, bile noire (Jouanna 2002). La théorie des humeurs et des tempéraments n'est cependant pas arrivée à son expression définitive avec Galien (Jouanna 2013: 313-334; 335-360). Elle se ramifie, se complète et se complexifie pendant la période médiévale et au sein de la civilisation arabo-islamique.

Il y a trois échelles de mélange: les éléments, les qualités et les humeurs (Ullmann 1995: 66-70). Selon Galien et pour les médecins médiévaux après lui tel que Rāzī (Iskandar 1961: 23-244; Moubachir 1996: 55, 56), chaque espèce animale possède son propre tempérament ou mizäğ en arabe (le verbe trilittère mazăga en Arabe signifie "mélanger»; Kazimirski 1860: 1098, B). Cette idée forme la base du chapitre III consacré aux animaux sauvages. Le meilleur mizäğ est celui de l'homme (Debru 2013) et le tempérament de chaque individu a un niveau de variabilité que le médecin doit connaître. Les conditions de vie, climatiques, géographiques, et les comportements apparents sont des signes pour découvrir le tempérament de chaque espèce et des individus au sein d'une espèce donnée (Boudon-Millot 2006: 130-132). Ces théories associées aux observations forment les bases des chapitres I (hommes et géographie) et III de l'ouvrage d'IbnAA sur la classification des animaux sauvages selon l'espace occupé. 
CONDITIONS DE VIE, CONDITIONS CLIMATIQUES.

Dans le traité hippocratique Airs, eaux, lieux, l'auteur tente de trouver dans les conditions climatiques et les saisons, les causes de la diversité des caractères (et des coutumes) des peuples sur la Terre, mais aussi les causes de l'apparition des maladies (Jouanna 2013). Six siècles plus tard, Galien rédige un commentaire au traité Airs, eaux, lieux, dont nous est parvenue une traduction arabe (Sezgin 2001). Dans ce texte, Galien conserve certaines tendances d'Hippocrate et s'écarte d'autres, mais conserve cette idée fondamentale de la théorie des climats. Les médecins et géographes médiévaux discutent de ces divisions de la terre habitée en climats (iqlìm, littéralement "zones, régions» en arabe) selon leur propre expérience et leur propre pratique (Katouzian-Safadi \& Ben Saad 2019: 220, 221). Pour exemple, nous pouvons rappeler l'opposition entre deux médecins, Ibn Ğazzār († 979) et Ibn Riḍwān († 1061) quant à leur interprétation des maladies des habitants du Caire et de sa banlieue. Les propos d'Ibn Riḍwān sont basés sur ses observations climatiques directes de l'Égypte. Mais Ibn Ğazzār n'avait jamais vécu en Égypte et son raisonnement était basé sur les propos rapportés par ses compatriotes. (Gamal \& Dol 1984: 12 [texte arabe] ; 99 [traduction anglaise]). Le chapitre III sur les animaux sauvages s'appuie sur cette relation entre intérieur/extérieur, bien connue des médecins: "tempérament», humeurs, quatre qualités et quatre éléments/milieu de vie.

\section{PhysiognOmie}

Le tempérament de chacun peut avoir des effets sur l'ensemble de la forme corporelle et des comportements apparents d'un individu, comme par exemple sa corpulence, la forme des diverses parties de son visage, la rapidité ou la lenteur dans ses mouvements, sa voix et aussi sur ses capacités morales. La physiognomonie doit permettre d'accéder aux tempéraments et aux caractères moraux à partir de la connaissance de certains signes extérieurs (Wilgaux 2008) ou firāsa en Arabe (Siddīqi 1987: 79-107). Elle est répandue chez les savants et particulièrement chez les médecins quil l'utilisent pour découvrir le tempérament propre à chaque individu, ses qualités morales et ses capacités particulières de travail (nourrice, serviteur, chanteur, comptable...). La physiognomonie a pu être appliquée pour proposer des critères de sélection pour le recrutement du personnel au service d'un maître dans des traités portant sur l'achat des esclaves (Sanagustin 2010). IbnAA ne cite pas le terme firāsa dans les fragments de son traité que nous avons analysés; néanmoins, il établit en permanence une relation entre la composition interne des animaux (qualité des éléments qui les composent, leur tempérament) et la rapidité, la lenteur, lors de la marche ou de l'envol; la classe des «animaux Air - Feu » est dotée d'une rapidité de vol, grâce aux qualités légères de leurs constituants. Il déduit également des comportements moraux comme la douceur ou au contraire l'agressivité et la dureté chez la classe des " animaux Terre-Feu ", l'élément Terre étant sec et froid et l'élément Feu facilitant le mouvement par sa légèreté. Ces théories créent ainsi une interdépendance entre tous ces principes des plus simples aux plus complexes: éléments supports des qualités, humeur, tempérament, physiognomonie, comportement, caractères moraux.
TABLE 1. - Translittération des caractères arabes en caractères latins.

\begin{tabular}{|c|c|}
\hline Arabe & Translittération internationale \\
\hline أ & , \\
\hline 1 & $\overline{\mathrm{a}}$ \\
\hline ث & $\underline{t}(\mathrm{c})$ \\
\hline ج & $\breve{g}$ \\
\hline$\tau$ & ḥ \\
\hline$\dot{\tau}$ & b \\
\hline$\dot{j}$ & $\mathrm{~d}$ \\
\hline 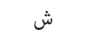 & š \\
\hline ص & ș \\
\hline ض & d \\
\hline$b$ & ț \\
\hline ظ & $z$ \\
\hline$\varepsilon$ & $i$ \\
\hline$\dot{\varepsilon}$ & $\dot{g}$ \\
\hline ق & $\mathrm{q}$ \\
\hline و & $\bar{u} / w$ \\
\hline ي & $\overline{1} / \mathrm{y}$ \\
\hline
\end{tabular}

\section{PHARMACOPÉE, CLASSIFICATION}

Dans le domaine pharmaceutique, la description d'un produit donné (Katouzian-Safadi 2004a; Katouzian-Safadi \&Karamati 2018: 30, 31) commence d'abord par l'indication de ses qualités (chaud, froid, humide ou sec) assortie de celle de leur degré (Siddīqī 1987: 189-194). Dans le fragment sur les animaux «sauvages» que nous examinons ci-dessous, IbnAA, influencé par sa formation médicale, adopte une convention de classification selon les éléments (porteurs de qualité) dominants des produits, classification fonctionnelle et courante chez les pharmaciens et médecins médiévaux.

\section{LES DEGRÉS DES QUALITÉS}

Galien avait divisé en quatre degrés d'intensité chacune des quatre qualités que sont le chaud, le froid, le sec et l'humide (Galien, De simpl. Med. Per. Fac. 1.27 (11.428-430 K.), 3.10 (11.560-561 K.), 5.7 (11.786-788K); De comp. Med. Per gen. 1. (13.367-9 K.); Debru 1997). Originellement séparés par une progression arithmétique de un (Debru 1997: 85-102), al-Kindī († 873) propose par la suite une progression géométrique (Stephan 2002) de raison deux et applique ensuite cette théorie pour la première fois aux médicaments composés (Stephan 1999). Sous cette forme, celle-ci est acceptée par un cercle restreint de pharmaciens (Deboth Ricordel 2004, Ricordel 2011), de médecins et de savants en Orient ou en Occident, mais fortement rejetée par d'autres comme Ibn Rušd/Averroès († 1198) (Stephan 2011), tandis que certains l'ignorent tout simplement.

Remke Kruk (2005) a démontré l'approche diététique adoptée par IbnAA au sujet des animaux domestiques au deuxième chapitre de son Kitāb al-H (Kruk 2005). Au sein du corpus diététique il est courant d'indiquer les degrés des qualités. IbnAA applique aux aliments la forme courante proposée par Galien, où les degrés vont de un à quatre. Par exemple dans le cas de lahm (pluriel luḥum et que nous désignons ci-dessous par la «chair animale» sous forme crue ou «viande» sous forme consommée par l'homme), IbnAA écrit d'une certaine viande de chèvre (lahm al-ma'az) qu'elle est "humide à l'extrême du premier degré de l'humidité» (rațb fì āhir al-dară̆a al-ūlā); 
il conçoit aussi l'existence des moitiés de degré, "humide d'une humidité d'un demi-degré parmi les degrés" (rațiban fì niṣf al-dară̆a min al-dară̆a; Harbī 2008: 189, 1. 15, 16) mais émet des doutes quant à la validité de l'appréciation et à la précision des degrés des qualités. Pour lui, l'existence des qualités propres aux quatre éléments est une propriété ('ilm, littéralement un «savoir») valide comme lorsqu'en géométrie on dit que la somme des angles d'un triangle (mutalat) équivaut à deux angles droits (musāwiyatayn al-qäimatayn): Le feu est chaud (härra), brûlant (muhriqa) et sec (mü̆affifa), l'eau est froide ( $b \bar{a} r i d)$ et humide ( $r a t+b)$, l'air est chaud et humide et la terre est froide et asséchante (yābisa) (Harbī 2008: 189, 1. 4-11). Mais il n'en est pas de même lorsque l'on désigne une viande (lahm) comme étant chaude au premier degré: dans ce cas, toujours selon IbnAA, la médecine procède par supposition (hads) et par approximation (taqrīb) (Harbī 2008: $153,1.5,6)$. Ces précisions révèlent sa formation médicale théorique autant que son souci pratique de thérapeute pour quantifier les propriétés des aliments.

Souligner comme il le fait que toutes les connaissances en médecine n'atteignent pas le même degré d'exactitude, indique une réflexion approfondie sur sa propre science.

\section{LES PROPORTIONS DES ÉLÉMENTS}

La théorie des quatre éléments a donné d'autres extensions théoriques. Auteur ou fondateur d'une école de pensée très originale sur l'alchimie arabe, Ğābir Ibn Haywān (Kraus 1986: 38, 40, 41, 173; 1986) a concentré ses efforts dans la purification de la matière par distillation (Katouzian-Safadi 2014: 17), pour purifier le plus possible les "éléments» et fabriquer d'autres corps composés à partir d'eux. Il pensait, par l'analyse des matériaux, parvenir à expliquer toutes leurs propriétés spécifiques. Rāzī et Ibn Sīnā - dit Avicenne acceptaient que les particularités de chaque produit proviennent de leur composition et du rapport entre les quatre éléments et les quatre qualités présentes dans chaque corps, mais considéraient que la connaissance de ce rapport précis demeurait inaccessible à l'homme (Katouzian-Safadi 2004b). Pour IbnAA, les quantités et les proportions de chacun des quatre éléments présents déterminent la classification et le comportement des animaux sauvages, comme nous le verrons ensuite.

Quoique les divers domaines de savoir (géographie, médecine, pharmacie...), comme les auteurs cités précédemment, appartiennent à des écoles de pensée différentes, il est possible d'observer des points communs dans la démarche de mise en relation entre environnement, comportement des animaux ou des plantes et nature ou composition interne supposée et déduite. Ce cheminement et ces points sont rassemblés et représentés dans la Figure 1. Les quatre niveaux A, B, C, D, sont en relation et peuvent être situés sur un cercle marquant leur interdépendance. Cependant il est essentiel de souligner que ce schéma général laisse la liberté à chaque savant de développer ses propres idées, conceptions et visions du monde. C'est dans ce cadre qu'IbnAA développe une vision originale de la classification des animaux «sauvages". Dans ce qui suit, nous proposerons un court aperçu de ces principes, avant IbnAA, et nous exposerons l'économie de la division en chapitres de son Kitāb al-H (Harbī 2008). Il s'agit principalement d'examiner le cas des animaux «sauvages » en replaçant ces réflexions dans le cadre général des théories citées au cours de cette section.

\section{DIVERS PROCÉDÉS DE CLASSIFICATION DES ANIMAUX CHEZ LES AUTEURS ARABES AU MOYEN ÂGE}

Toutes les communautés humaines anciennes ayant vécu avec les animaux ont été concernées par la classification des animaux. Les classifications médiévales sont basées sur les savoirs locaux oraux, sur les lectures des textes des philosophes, des savants et des médecins indiens, persans, arabes ou grecs. Ces écrits zoologiques ont été particulièrement marqués par certains écrits d'Aristote (IVe siècle av. J.-C.) et de Galien († 216) qui ont circulé en grec et en syriaque, avant (Hugonnard-Roche 1997) et après l'avènement de l'Islam (Le Coz 2004), au Proche-Orient où ils ont été traduits en arabe à plusieurs reprises. C'est avec Hunayn Ibn Ishāq et ses disciples (Gutas 1998) que les traductions des œuvres de Galien prennent une forme savante. Trois œuvres zoologiques d'Aristote, l'Histoire des animaux, les Parties des animaux et la Génération des animaux, ont été rassemblées et traduites en Arabe pour donner le Livre des Animaux (Kruk 1979; Filius 2018). Dans son Histoire des animaux, Aristote a classé les animaux en sélectionnant différents critères. Cette classification a été examinée et analysée. Des études anciennes et décontextualisées considéraient que la classification d'Aristote était incomplète et flottante (Manquat 1932); d'autres interprétations " continuistes» cherchant les esquisses de théories futures ont tenté de l'apparenter à une classification plus moderne (Bourgey 1955). Des recherches plus récentes, historiennes et contextualisées, ont permis de mieux rendre compte de l'importance de l'œuvre d'Aristote au sein de l'histoire de la zoologie (Zucker 2005). Divers articles de ce volume illustrent ce point. Nous nous sommes inspirés de ces travaux et il ne nous semble pas nécessaire de les évoquer ici, cela ayant été fait profondément et n'étant pas l'objet de notre étude. De nombreuses traductions françaises de l'Histoire des Animaux d'Aristote sont disponibles (Pellegrin 1982). Cette ouvre une fois traduite, a eu une influence majeure sur les savants médiévaux de langue arabe qui ont considéré divers critères pour définir les catégories animales. Parmi les quelques exemples détaillés ci-dessous, portant sur les régimes alimentaires, nous constaterons que certains sont basés sur des critères perceptibles à l'œil (mouvement, génération) et d'autres sur des élaborations conceptuelles, comme les éléments et leurs qualités. L'essentiel du contenu de ces corpus s'organise autour des catégories de viandes couramment consommables, c'est-à-dire les oiseaux, les quadrupèdes, les poissons et leurs produits dérivés. C'est ainsi que procède par exemple Rāzī dans son livre sur les qualités et les méfaits des aliments (Itani 1984). Les exemples ci-dessous sont sélectionnés pour illustrer nos propos et sont très loin de couvrir l'ensemble des modes de classification. 
A Observation d'un objet

- milieu d'existence (environnement vital)

- comportement des animaux

- qualités apparentes des produits de la pharmacopée

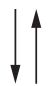

D Déduction de la nature des qualités dominantes de l'objet observé (animal ou produit médicamenteux) : éléments, quantité, proportion

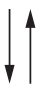

B Analyse du comportement d'un animal ou analyse des effets d'un produit médicamenteux sur les corps
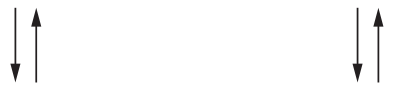

C Déduction du tempérament de l'animal ou d'un produit médicamenteux

FIG. 1. - Schématisation de la démarche d'observation: mise en relation établie entre les facteurs extérieurs et le tempérament des humains, des animaux ou des plantes. A, B, C, D, étapes successives de cette démarche.

\section{LE MODE DE DÉPLACEMENT}

Chez les auteurs arabes, l'un des savants à avoir mis par écrit la question de la classification de tous les animaux fut al-Ğāhị ${ }^{3}$ (776-868), polygraphe de Bassora. Son ouvrage Kitāb al-Hayawān (Hārūn 1996), qui porte le même titre que le Livre des Animaux d'Aristote, fut longtemps étudié exclusivement d'un point de vue littéraire, mais suscite depuis quelques années l'intérêt d'historiens de la biologie (Aarab 2001; Aarab et al. 2003; Ben Saad 2010; Ben Saad \& Katouzian-Safadi 2011; Aarab \& Provençal 2013; Chebbi Lamouchi \& Katouzian-Safadi 2013; Ben Saad et al. 2013; Aarab \& Lherminier 2015; Aarab \& Provençal 2016; Aarab 2017; Ben Saad 2017). Gāhiz adopte comme premier critère de classification le mode de déplacement des animaux, divisés par lui en quatre grands groupes: ceux qui marchent, ceux qui volent, ceux qui nagent et ceux qui rampent. Cette première division est discutée par l'auteur tout au long de son ouvrage, mais sa classification est quelque peu modifiée en fonction de ses observations. À ce niveau, il reprend en partie d'anciennes classifications anté islamiques. En effet, Gāhiz fait souvent référence aux grammairiens arabes des VIIIe et XIe siècles comme al-'Așma '́i (740-828) ou Abū 'Ubayda (770-838). Ces derniers avaient collecté auprès des habitants de la péninsule arabique, des informations sur la nomenclature et la vie des animaux qui révèlent une riche tradition de connaissance sur ces derniers, bien avant l'avènement de l'Islam (Aarab 2001). Les poèmes relayés dans le volume concernant la vie des animaux mettent en évidence cet héritage préislamique. Pour définir certains sous-groupes, Ğāhiz emploie d'autres critères comme l'alimentation, la morphologie ou des critères éco-

3. Al-Ğāhị sera nommé dans la suite de l'article, Ğāhị, dépourvu de l'article "al-» de la langue arabe. éthologiques (Chebbi Lamouchi \& Katouzian-Safadi 2013; Ben Saad et al. 2013). Beaucoup d'auteurs après Gāhịiz se sont référés à sa classification des animaux; citons par exemple al-Qazwīnī (1203-1283) dans son 'Ǎ̌āiib al-mahlūqāt wa garāì al-mawğūdāt (Qāọī Ben Yūsuf 2006) et al-Damīrī (1341-1405) dans Hayāt al-ḥayawān al-kubrā (Basaj 2007).

\section{LA GÉNÉRATION}

Les Ihwān al-Ṣafā’, souvent appelés en français les «Frères de la pureté », philosophes et savants arabes ismaélites du Xe siècle ayant vécu à Bassora, sont à l'origine d'une œuvre majeure, les Rasà'il Ihwān al-Ṣafä' wa Hillān al-wafă'. Deux épîtres y sont consacrées aux animaux, adoptant l'une et l'autre des points de vue très différents sur l'univers animal. L'épitre 22, discutée dans le présent volume (Vaulx d'Arcy comm. pers.), est un Miroir des Princes (Dakhlia 2002; Fouchecour 2010). Dans ce genre de récit, les animaux vivent en société comme les hommes et le but est d'enseigner à chacun comment se comporter selon sa position sociale pour avoir la vie sauve. Des aspects plus strictement zoologiques sont traités au sein de la huitième épître, où les auteurs introduisent un critère pour la classification des animaux en se basant sur la notion de génération spontanée (Kruk 1990; voir aussi, pour la génération spontanée traitée par Ğāhiz, Aarab 2001; Lamouchi Chebbi 2018: 167-176). Ils divisent les animaux entre ceux qui sont de "nature achevée " (tām al-hilqa), caractérisés par la copulation, la gestation, l'accouchement et l'allaitement, et ceux de «nature inachevée» (näqiș al-hilqa), comme ceux qui naissent dans la pourriture. Entre les deux se trouvent les animaux qui pondent et couvent (Bustānī 1957: 181). Les animaux de "nature inachevée" auraient existé chronologiquement avant les animaux de "nature achevée» car ils nécessitent moins de temps pour se former (Bustānī 1957:181). 


\section{LES ÉLÉMENTS ET LEUR DENSITÉ}

Pour établir les bases de sa théorie pharmacologique, Galien faisait appel à une série de notions basées sur les concepts aristotéliciens, en particulier celles de "léger», "ténu» (en grec, leptomère [Debru $1997^{4}$ ] et en arabe raqiq et latîff), et son opposé «lourd», épais, dense, (pachymère en grec et $\dot{g} a l i \hat{z}$ en arabe). Ces notions sont importantes en pharmacie, en diététique et alchimie (Katouzian-Safadi 2004b) et se retrouvent également très tôt dans les textes médicaux.

Le médecin 'Alī Ibn Rabbān Ṭabarī (mort après 853) décrit ainsi dans son ouvrage de médecine Firdaws al-Hikma une classification des animaux basée sur les éléments (Siddīqī 1928: 27-30) et sur leur densité. Il distingue trois genres d'animaux: de Terre ('ardì), de l'Eau (mầे), de l'Air (hawāì). Le feu et l'air sont légers (hafiff) alors que l'eau et la terre sont lourds ( $\left.\operatorname{taq}_{\bar{i}}\right)$. Ces divisions, appliquées par Țabarī à tous les animaux, domestiques ou sauvages, sont semblables à celles présentées dans le chapitre III d'IbnAA pour les animaux sauvages; de tels choix de classification existaient donc dans le monde médical avant IbnAA.

Un autre médecin, Ishāq Ibn Sulaymān al-Isrāàilì (845-950), s'est fondé sur les mêmes concepts pour la classification des animaux. Contemporain d'Ibn AA († 970), cet auteur en est cependant très éloigné géographiquement: Ishāq Ibn Sulaymān al-Isrāìilī 5 est un médecin et philosophe né en Égypte mais installé à Kairouan au début du $\mathrm{X}^{\mathrm{e}}$ siècle; ces territoires étaient alors sous le contrôle du califat fatimide (909-1171). Il est l'auteur d'un ouvrage sur l'alimentation et le régime alimentaire, le Kitāb al-adwiyah al-mufradah wa al-aǵdiyah (Șabbah 1992), où il se fait l'écho des théories des "Anciens naturalistes » ou tabi'zy yun, selon lesquelles les proportions des quatre éléments (Air, Feu, Eau et Terre) dans la constitution des êtres vivants définissent leur taille, leur régime alimentaire et leur mode de déplacement. Isrāîlī incorpore également aux éléments la notion de densité (raqīq et lațîfévoqués plus haut), ainsi que l'espace vital (air, terre, eau) et le mode de déplacement des animaux pour en réaliser une classification (Șabbah 1992: 83, 1. 22, 23; 84, 1. 1-11.; Dahrouch 2019: 172):

- le Feu et l'Air sont légers et forment les deux éléments qui se meuvent vers le haut ('unșurān hafîfän yataharrakān $\left.s a^{\prime} d a n\right)$; l'envol des oiseaux est associé à cette catégorie; ce sont les animaux aériens (volant dans l'air ou hawāà, țayyār); - l'Eau et la Terre sont lourdes et forment les deux éléments qui se meuvent vers le bas ('unșurān țaqìlān yataharrakān suflan); la marche des animaux terrestres est associée à ce groupe et ils sont donc dénommés ardī ou mašš $\bar{a}$, "marchant sur terre»; - entre les deux se trouvent les animaux qui nagent dans l'eau $(m \bar{a} \bar{\imath}, s \bar{a} b i h)$.

Il faut cependant noter qu'après cette courte introduction, l'auteur ne discute plus des quatre éléments, des modes de vie ou des comportements des animaux; il se concentre uniquement sur les notions liées à la diététique, même si Isrāîlī évoque également des animaux inhabituels pour la consommation comme l'ours, le lion, le renard. IbnAA

4. Debru indique que c'est dans le prologue De simplicium medicamentoeum temperamentis ac facultatibus que Galien développe ces points.

5. Cet auteur est nommé Isräīli dans la suite de l'article. quant à lui discute de ces notions de lourdeur et de légèreté au chapitre II, pour les animaux domestiqués dont la chair est consommée par l'homme.

La classification d'IbnAA a ses spécificités. En particulier, cet auteur applique le caractère constitutif des éléments comme critère de séparation uniquement aux animaux "sauvages » et non pas aux animaux comestibles «domptés par l'homme». Ensuite, IbnAA détermine une classe d'animaux de Feu pur, sans mélange avec d'autres éléments. Enfin, au sein de chaque paragraphe réservé à une classe d'animaux sauvages, IbnAA souligne le rapport entre les éléments constitutifs du corps de l'animal, son lieu de vie, son mode de déplacement et l'ensemble de ses comportements physiques et moraux; son regard est plus proche des zoologistes.

\section{LA CLASSIFICATION DES ANIMAUX PAR IBN ABĪ AL-AŠS'AT ET SON CEUVRE KITĀB AL-HAYAWĀN}

L'auteur décrit lui-même son ouvrage comme suit:

«Mon livre est à la fois un traité sur la nature $\left(\operatorname{tab}^{\prime} a\right)$ et la médecine $(t i b b)$; il est aussi un traité de métaphysique ( $\left.m \bar{a} b a^{\prime} d a l-t a b \bar{i}^{\prime} a\right)$ car quand je parle de l'âme humaine (nafs al-insān), c'est de la métaphysique et si je parle de l'âme de l'animal (nafs al-hayawān) et de son ressenti (ihssāsihi), c'est de la nature et quand je parle de son alimentation ( $m \bar{a}$ yugitad $\bar{a}$ bihi) c'est de la médecine. » (Harbī 2008: 153)

Pour cette classification des êtres vivants hayawān, IbnAA les définit ainsi : les humains (hay mālik), les animaux domestiques ( hay mamlūk) et les animaux sauvages (lā mälik wa là mamlūk). Le verbe malaka en arabe désigne l'action de posséder, les humains sont donc ceux qui "possèdent", l'auteur les désigne aussi par le terme al-'aql (la raison). Les animaux domestiques sont ceux "possédés" par les humains, et les animaux sauvages ne sont ni l'un, ni l'autre. Kruk (2001) utilise pour traduire toutes ces notions les termes «ruler" pour les humains, « ruled» pour les animaux domestiques et «neither ruler nor is ruled» pour les animaux sauvages. Dans la suite de notre travail, nous utiliserons les termes «domestiques» et «sauvages » pour signifier respectivement mamlūk et là mālik wa là mamlük.

\section{LA RAISON HUMAINE}

Dans la pensée philosophique médiévale, dès le savant al-Kindī († 873), ce terme ' $a q l$, «la raison» (ou encore «l'intellect» selon les champs et débats philosophiques) a connu divers développements (Jolivet 1971; Jolivet \& Rached 1998). Très tôt, les philosophes et les savants se basent sur la notion de raison humaine ou 'aql (terme usité pour traduire le terme Grec noûs) pour distinguer l'homme de l'animal. La raison humaine, cette source pour découvrir le monde, a été considérée comme une faveur et une supériorité accordées à l'homme par rapport à l'animal. Ainsi, dans le premier chapitre de son livre Kitāb al-tibb al-ruhānī (Kraus 1939: 17, 18) ou La médecine spirituelle (Saad 2002; Brague 2003: 56, 57), Rāzī écrit: 
«Elle [la raison, 'aql] est la plus grande grâce de Dieu envers nous, la chose qui nous est la plus utile et la plus avantageuse. L'intellect nous rend supérieurs aux animaux dépourvus de raison si bien que nous les possédons, les gouvernons, les soumettons et les utilisons de telle sorte qu’il en résulte des bénéfices pour eux et pour nous [...] Elle [la raison, ' $a q l$ ] est la chose sans laquelle notre condition (hâla) serait celle des bêtes quadrupèdes herbivores (albaha'im $\left.{ }^{6}\right)$, des enfants (al-attfăl) et des fous (al-măgāninn).»

Les discussions sur les animaux, sur leur utilité pour l'homme, sur le degré de souffrance que l'homme pouvait leur infliger, ou sur le droit de les tuer ou non, faisaient partie des débats entre philosophes, savants et penseurs du Kalām (théologie rationnelle en Islam), dans lesquels Rāzī était très engagé (Rashed 2000).

Dans son livre Kitāb al-sīra al-falsafìyya (Livre de la vie philosophique), Rāzī ne s'intéresse pas aux animaux dans une perspective de classification zoologique ${ }^{7}$, mais souhaite plutôt clarifier comment l'homme doit se comporter face à eux (Adamson 2012: 260-271). C'est dans cette perspective qu'il considère trois catégories distinctes d'animaux (Kraus 1939: 104, 1. 16-19):

- ceux qui consomment de la chair animale comme le lion ('asad), la panthère (namir) et les loups $\left(\underline{d} i^{\prime} \bar{a} b\right)$;

- les animaux nuisibles et dont l'homme n'a pas besoin, comme les serpents (hayyāt), les scorpions ('aqārib); Rāzī ( Kraus 1939: 105, 1. 8) tolère leur destruction par l'homme ( $\breve{g} \bar{a} z a$ iblākuhā); - les animaux gouvernés par l'homme (Kraus 1939: 104, 1. 16-19) (al-haywān al-ma'müla) et qui vivent de l'herbe (al-'àiša bi al-'ušub). Rāzī les décrit comme des animaux que l'homme consomme ou utilise comme monture. Avec ces animaux, l'homme doit se comporter avec douceur (rifq).

Le point commun de cette classification avec celle d'IbnAA réside dans le fait que les animaux utiles à l'homme sont séparés des animaux «sauvages », même si Razī utilise sa propre terminologie. Il faut noter que le mode d'alimentation des animaux est également présent dans les classifications de Rāzī et d'IbnAA.

En tant que zoologiste, Gāḥiz se distingue de ces deux auteurs; l'environnement animal et la santé de l'homme sont deux de ses sujets de réflexion (Aarab et al. 2019), tandis que le caractère utile ou nuisible n'intervient pas dans sa classification des animaux (Aarab 2001; Ben Saad 2010). Dans son Kitāb al-Hayawān, al-Ğāhị (Hārūn 1996: III, 394) rapporte les propos d'un maître $m$ 'tazilite $^{8}$, al-Naẓz̄ām, né presque un siècle avant Rāzī et mort au milieu du IXe siècle. Al-Naẓāām

6. Le terme exact pour traduire al-baha'im est selon Ahmad Aarab (2001) les animaux herbivores.

7. Druart (1996) et Adamson (2012) exposent leurs thèses opposées quant aux objectifs de ce débat philosophique de Rāzī. Le débat concerne la justice divine et la destinée de l'âme chez les humains et les animaux; nous n'exposons pas ces thèses dans le cadre de notre article.

8. Le mu'tazilisme est l'une des écoles de pensée rationnelle théologique et philosophique de l'Islam, voir l'Encyclopédie de l'Islam vol. VII (Bosworth et al. 1993: 783-793). Ğāhiz était un des maîtres de ce courant qui associait à toute recherche, le raisonnement et la rationalité. La richesse de cette école théologique et philosophique provient de l'existence de grands nombres de courants internes et des débats sur des sujets philosophique comme l'atomisme, la création, le libre arbitre (Rashed 2008). pense qu'il n'y a pas de différence entre les enfants, les bêtes quadrupèdes herbivores et les fous, termes précis que Rāzì utilisera plus tard. Rāzī avait probablement connaissance de ces textes et de ces discussions; ses positions sur la relation des humains avec les animaux sont en tout cas imprégnées de ces enseignements (Adamson 2012: 253). Cette distinction première entre l'homme et les autres animaux, basée sur la raison humaine, était donc une idée partagée entre de nombreux philosophes et savants médiévaux. Chez IbnAA, la Raison devient la clef de la voûte de la classification et la base de la division des vivants considérés.

\section{CHAPITRE I: LES HUMAINS - CHAPITRE II: LES ANIMAUX DOMESTIQUES}

Nous rappelons que cet ouvrage est divisé en trois chapitres (trois maqāla) consacrés respectivement aux humains (presque $45 \%$ du contenu), aux animaux domestiques (33\%) et aux animaux sauvages (22\%).

\section{CHAPITRE I : LES HUMAINS, HAY MĀLIK}

$\mathrm{Au}$ chapitre premier, IbnAA développe sa conception de la théorie des espaces géographiques, iqlim (climats), en relation avec les tempéraments des humains occupant ces régions. Au lieu de parler d'Iqlīm, IbnAA parlera de maskan' (masakin au pluriel), l' "habitat" (Kruk 2001: 128, 1. 3). Nous retrouvons ce terme dans le traité Fì al-hawā wa al-mā wa al-masākin qui est la traduction arabe du commentaire au traité d'Hippocrate Air, eaux, lieux (Sezgin 2001). IbnAA discute de l'habitat des humains et affine son approche en tenant compte de l'espace, ville, campagne, montagne, bord de mer, vallée... Ce chapitre dépasse de loin en volume et en précision ce qu'habituellement le corpus des livres de médecine réserve à la géographie humaine. IbnAA affirme que, selon la région habitée, les humains diffèrent par l'allure, la morale, l'humeur, la couleur, la sagesse, l'ignorance, le courage, la peur, la force et la faiblesse (Harbī 2008: 29). Le chapitre I a donc pour vocation de permettre aux médecins de définir les tempéraments des humains pour proposer à chacun un régime alimentaire adapté.

\section{CHAPITRE II: LES ANIMAUX DOMESTIQUES : HAYY MAMLŪK}

Pour IbnAA, les animaux domestiques (Kruk 2001: 135145 ; 2005: 93-101) sont gouvernés par la nature et par la raison ( $a l-' a q l)$ humaine, car l'homme intervient dans leur existence (wuğḡd), leur survie $(b a q \bar{a})$ et dans leur multiplication par génération ${ }^{10}$ (tawāllud) (Harbī 2008: 149); ces interventions humaines établissent une relation d'asservissement. Mais IbnAA va plus loin; pour lui, notre Créateur (häliqunā) a conçu ces animaux afin qu'ils soient dominés et que leurs bénéfices (manāfi) profitent à l'humain. Selon lui, la domestication n'est donc pas le fruit historique de la raison et l'effort humains.

9. La racine trilitère du nom de lieu maskan, s k n, désigne la demeure (Kazimirski-1860: 1116, A). Le terme iqlìm désigne un espace géographique.

10. Autrement dit, reproduction. 
Ce groupe se divise ensuite en quatre sous-groupes selon leur utilité pour l'homme. Nous trouvons ainsi l'animal monture, l'animal nourriture, l'animal à la fois monture et nourriture et enfin, l'animal domestiqué au service de l'homme ou mum$\operatorname{tahan}^{11}$, comme le chien et le chat.

Au sujet des montures, l'auteur prend uniquement l'exemple des chevaux (hayl), des mulets (big $\bar{a} l)$ et des ânes (hamīr). Il décrit leurs différentes espèces, compare leurs caractéristiques physiques et "morales» en liaison avec leur qualité comme monture et aborde aussi des questions relatives à leur reproduction.

Comme l'a montré R. Kruk (2005), une partie du chapitre II donne des indications diététiques. Pour les animaux consommables, IbnAA explique qu'ils auraient une complexion (un tempérament) ou mizāğ proche de celle des végétaux consommables par les humains. Il faut rapprocher ces propos d'IbnAA du fait que ces animaux ne sont pas carnivores mais herbivores en majorité. Il prend comme exemple le mouton (harūf), la chèvre (ma'z), le porc (hinzīir), la poule (dağḡ $\breve{g})$, le pigeon domestique (hamàm ahlì) et le canard domestique (batt ahlì). De longs passages sont ainsi consacrés aux produits dérivés de ces animaux comme les viandes (luhūm), les œufs (bayd) et les laitages (laban, ğubn, zubd) et l'impact de leur consommation sur la santé et la complexion humaine. L'auteur s'intéresse plus à la diététique qu’à la classification. Le médecin doit mettre en relation ces qualités diététiques avec les divers tempéraments des humains selon leur espace de vie (chapitre I) pour définir le bon régime alimentaire pour son patient.

Dans le cas des animaux à la fois monture et nourriture, IbnAA donne uniquement deux exemples: le chameau ( ğamal) et la vache (baqar). La vache ne pouvant pas être montée directement, elle sert néanmoins, dans certaines régions d'Iraq, à transporter des charges lourdes, écrit-il. Enfin, dans la quatrième catégorie, celle de l'animal mumtahan, nous trouvons le chat (hirr) et chien (kalb). Les deux partagent la particularité de s'attacher aux humains et de leur être utiles. Le premier traque les nuisibles (hayawānāt mu'dìyya) et le second peut être un bon gardien et être utilisé pour la chasse (sayd). Les «bénéfices» (manāfi) de ces animaux, signalés dans l'introduction de son œuvre, sont ainsi justifiés par IbnAA.

\section{CHAPITRE III: LES ANIMAUX SAUVAGES, $L \bar{A} M \bar{A} L I K W A$ LA MAMLÜK}

IbnAA consacre le troisième maqāla de son livre, qui nous intéresse tout particulièrement ici, aux animaux sauvages. Sa démarche dans la classification des animaux sauvages rappelle celle de Ṭabarī ainsi que celle d'Isḥāq al-Isrāīlī pour classer les aliments utiles à l'homme (voir le paragraphe «Les éléments et leur densité»). Mais la ressemblance s’arrête là car IbnAA associe à cette classification un regard et des réflexions élaborés de zoologiste. IbnAA définit seize catégories animales:

11. La racine est $m h n$; le verbe mahana signifie servir quelqu'un; en Arabe, la forme verbale VIII (imtahana) signifie se faire domestique ou entrer au service de quelqu'un comme un valet (Kazimirski 1860: 1163, B.) quatre «simples» et douze "composées» (Harbī 2008: 242315). Cette classification sera reprise dans Mabähiğ al-fikr wa manāhiğ al-ibar de l'auteur et compilateur égyptien al-Wațāt (1235-1318) (pour quelques développements de la réception de Kitāb al-H d'IbnAA, voir Kruk 2001: 158-163).

Dans la première des sept sections $(b \bar{a} b)$ de ce chapitre, l'auteur expose les bases de sa classification des animaux sauvages à grand renfort d'exemples. Dans les sections suivantes, il entame des réflexions plus approfondies sur les différentes catégories et reprend ses exemples pour décrire leur nature avec beaucoup plus de minutie; cette maqāla ou chapitre III est cependant inachevée et n'aborde avec détail que six catégories sur les seize préalablement définies.

La classification des animaux differe dans les chapitres II et III. Dans ce dernier, IbnAA développe une "théorie » pour classer ce genre (ğins) d'animal qui, écrit-il, est gouverné uniquement par la nature $\left(t a b i^{-} a\right)$ dont son existence est dépendante; il est analogue en cela aux plantes sauvages (šăgar al-barrīyya wa al-ğabaliyya). Ces animaux, poursuit-il, fuient les humains car ils ont une peur instinctive ( $h \bar{a}^{\prime}$ if $b i$ al-tab $)$ d'être domptés et domestiqués. Le terme "manāfi $\tilde{i}^{\natural}$ ", les bénéfices, revient souvent à la forme négative pour rappeler qu'ils n'ont que peu d'utilité pour l'être humain. Cependant, écrit-il, certains animaux sauvages peuvent être utiles aux humains en leur fournissant de quoi se nourrir - comme la gazelle ( $\dot{g} a z a l)$, le francolin (durāğ), le poisson (samak) - et de quoi s'habiller - comme la zibeline (sammür), le pékan (dalaq), le fennec (fanak) et les panthères (numür) - ou encore en servant d'auxiliaires de chasse, et même comme preuve de la puissance de Dieu, tels les petits animaux nuisibles: les punaises (baq), les farās ${ }^{12}$, les fourmis (naml) et les araignées ('anākib) (Harbī 2008: 249).

Ibn abī al-Aš at explique le principe de sa classification en ces termes:

«Étant donné qu'il est reconnu (iqrār) que les principes du monde sont au nombre de quatre: le feu, l'air, l'eau et la terre, tous les êtres corruptibles sont formés à partir d'eux et reviennent à eux par corruption, et puisque l'animal (lā mālik wa lā mamlūk) est un être corruptible (mina al-kä ināt al-fäsidāt), alors il est composé de feu, d'air, d'eau et de terre. Ainsi cela conduit à plusieurs variétés (Aqsäman katīratan), par la nature (bi al-țab) et par le nombre à cause de ses différentes compositions (li-ihtiläf tarkỉbihi) à partir des quatre éléments et du penchant de la complexion (mayl al-mizāăg) de chaque genre vers un élément ou plus. L'animal (lā mālik wa là mamlūk) est: $\mathrm{a}^{13}$-A nature de feu ou b-Aérien ou c-Aquatique ou $\mathrm{d}$-Terrien. Ces quatre sont les natures les plus simples (absat al-țabäi'). Puis elles se composent [entre elles] (tatarakkab) et deviennent: a-De feu aérien ou b-Aérien de feu ou c-De feu aquatique ou d-Aquatique de feu ou e-De feu terrien ou f-Terrien de feu ou g-Aérien aquatique ou h-Aquatique aérien ou i-Aérien terrien ou j-Terrien

12. Désigne une catégorie d'insectes volants attirés par le feu ou la lumière (Lamouchi Chebbi, 2018: 66)

13. L'auteur utilise les lettres de l'alphabet arabe ( $a b \breve{g} a d$ ) pour énumérer les différentes classes d'animaux sauvages. 
aérien ou k-Aquatique terrien ou l-Terrien aquatique. Cela fait douze combinaisons qui sur la base de la dominance (al-tagalub) et la suprématie (al-tafädul) [d'un des composants] donnent douze natures $\left(t a b i^{i} a\right)$ pour douze genres (̆gins) et si on les ajoute aux quatre natures simples nous aurons au total seize natures pour les [différents] genres d'animaux (là mālik wa là mamlūk)» (Harbī 2008: 252).

Soucieux d'expliquer sa démarche et conscient du fait qu'un animal ne peut pas être composé d'un constituant unique ${ }^{14}$, il explique ce qu'il appelle une nature simple:

«[...] et puisque ces genres sont au nombre de seize, comprends alors que si je dis d'un animal qu'il est de feu, aquatique, aérien ou terrien, je lui donne le nom correspondant à l'élément le plus dominant dans sa constitution [...] car sinon cet animal serait simple et s'il était simple il agirait selon l'élément qui le constitue. L'animal de feu monterait vers le haut éternellement et ne pourrait pas descendre et cela est impossible, car s'il était de [nature ou de composition] simple (basiț $)$ il serait le feu [même]!» (Harbī 2008: 253)

Dans la même logique il explique la nature composée:

"Comprends aussi que si je dis animal de feu aquatique, cela signifie qu'il y a plus de feu que d'eau dans sa constitution et que sa nature de feu domine sa nature humide.»

La composition du corps de l'animal est donc en lien direct avec sa nature ( $\left.\operatorname{tab}^{-} a\right)$ et avec le milieu où il évolue. La nature de l'animal influence sa façon de se nourrir et lui donne les organes nécessaires (Harbī 2008: 254): plus un animal est chaud, par exemple, plus il a tendance à être carnivore. Les animaux les plus chauds se nourrissent d'animaux encore vivants, ceux qui sont moins chauds se nourrissent d'animaux qu'ils tuent euxmêmes et ceux qui sont encore moins chauds se nourrissent de cadavres, tels les oiseaux charognards. Cette grille de classification zoologique d'IbnAA est imprégnée par la tradition de la médecine et de la classification de la matière médicale dans les textes de pharmacopée. On peut évoquer par exemple le chapitre trois, sur les médicaments, du livre de Rāzì Kitāb al-Manșūrì (Siddīiī 1987: 189-194): les médicaments sont regroupés selon leur chaleur, leur froideur, leur sécheresse, leur humidité, leur degré de chaleur et de froideur, de sécheresse. D'autres domaines sont convoqués par IbnAA: la physiognomonie, dans la mesure où la chaleur est associée à l'état carnivore, à l'activité de la chasse ou à l'agressivité; la zoologie aussi est présente, car les organes sont associés à leur fonction et leur utilité.

Il est important de noter qu'IbnAA explique dans son texte qu'il n'examine que des espèces d'animaux qu'il connaît et ne détaille que ce qu'il a observé et expérimenté personnellement (Harbī 2008: 273). Cependant, il estime qu'il serait aisé

14. Dans son traité sur la connaissance de la force des médicaments composés (fì márifat quwwa al-awiya al-murakkaba), al-Kindī prend soin d'expliquer que chaque produit de la pharmacopée est formé des quatre éléments avec un ou deux éléments dominants (Stephan 2002: 213, 214). d'y insérer d'autres animaux qu'il ne connaît pas et invite à plusieurs reprises ses lecteurs à le faire. Exposée ainsi, l'auteur estime donc que cette classification est finie, complète et achevée et ne laisse pas de place à l'imprévu.

Après avoir expliqué sa démarche, IbnAA définit une à une les catégories dans la première section du chapitre III en donnant des exemples d'animaux et en commençant par les catégories simples.

\section{ANIMAL DE FEU}

L'animal de feu est de nature chaude et sèche, coléreux, rancunier, méchant, féroce et se nourrit d'animaux vivants. Il vole très haut au-dessus de l'air (chaud et humide), dans l'air chaud et sec de même composition que lui, et c'est cette même composition qui lui confère les organes adaptés pour atteindre ce milieu. Cet animal possède d'autres organes liés à son activité et à sa morale, écrit IbnAA, telles des serres et des pattes fortes pour attraper et transporter ses proies, ainsi qu'une acuité visuelle impressionnante. Il habite par nature les sommets des montagnes et les arbres les plus hauts. L'animal de feu préférerait la nourriture à sa propre liberté et c'est pour cela qu'il est possible de l'apprivoiser en lui procurant ses proies habituelles. Pour finir, l'auteur donne plusieurs exemples tels que l'aigle ('uqāb), l'épervier ( $b \bar{a} s ̌ i q)$, le faucon ( $b \bar{a} z \bar{i}$, terme d'origine persane), le milan (hada'a).

Dans le dernier chapitre de l'ouvrage, l'auteur reprend cette catégorie pour décrire avec beaucoup de détails la nature des animaux qu'il a pris comme exemples. L'aigle serait le rapace le plus «chaud» et dont la complexion est la plus sèche. De ce fait, sa chair est à consistance ferme et nerveuse (laḥm a 'ṣabì) et cela facilite son envol (Harbī 2008: 274).

Nous retrouvons ici comme dans la suite du texte, des passages qui rappellent la physiognomonie en médecine comme nous l'avons déjà décrite. La composition, le tempérament serait la source des caractères physiques (l'envol), moraux (coléreux, rancunier, féroce) et de certains comportements et habitudes (se nourrir d'animaux vivants).

\section{ANIMAL AÉRIEN}

Ce dernier, comme l'air, est de nature chaude et humide. Il vole dans la "vapeur" de la terre (au-dessus de l'eau) et la portée de son vol est courte, ce qui l'empêche d'être un oiseau migrateur. Cet animal habite les bosquets au bord de l'eau et de ce fait il est de complexion chaude et humide. Il se nourrit de graines et de baies et c'est pour cette raison que sa nature est proche de celle des humains et que sa viande convient au régime alimentaire humain. Perdrix ( $q a b a g$ ) ), faisans ( $t u d r u g$ ) et cailles (simmān) font partie de cette catégorie et IbnAA ajoute: "Si vous connaissez un animal similaire, associez-le à la nature de ce genre car je ne connais pas tous les animaux de la Terre» (Harbī 2008: 256).

Dans la troisième section $(b \bar{a} b)$ de ce troisième chapitre (maqāla), IbnAA revient à cette catégorie pour expliquer leur vol de basse altitude, dû selon lui au fait que les pennes de leurs ailes (qawādim) sont dirigées vers l'intérieur et que leurs ailes sont en forme de cuillères. Leurs ailes sont ainsi faites pour leur permettre de se déplacer dans un milieu «épais» ( $\dot{g} a l \bar{l} z$ ) comme la vapeur. 
ANIMAL AQUATIQUE

Il s'agit de tout animal qui meurt en quittant l'eau, "de ceux que nous connaissons ou pas" ajoute IbnAA. Il est de nature froide et humide avec différentes complexions possibles: ainsi celui vivant dans les fleuves (comme le Tigre) diffère de celui vivant dans les lacs ou l'eau salée. Pour comprendre cette différence, IbnAA énonce la loi «qānun» suivante:

"Ceux qui ont un corps lisse et sans écailles sont les plus froids, les plus humides, et la complexion est la plus dense. C'est pour cette raison qu'ils sont les plus gras, comme le poisson chat (al-ğarìt), l'anguille (al-marhāmì), le maquereau (al-kan'ab), le requin (al-küdinğ) et ceux qui leur ressemblent. Ceux qui ont les écailles les plus grandes, les plus nombreuses et les plus belles sont les plus chauds et les plus secs comme les poissons crocodile (al-sātirir) et ceux qui leur ressemblent. Ceux qui ont les écailles grandes, molles et peu nombreuses sont lisses, rapides et sauteurs. La couleur de leur dos et de leurs écailles tend vers le noir. Ce sont les plus chauds et les plus humides, comme la carpe (al-šabbüṭ), le sarü ' [non identifié], le hāznā [non identifié] et le barbeau (al-bunnì). Ceux qui ont les écailles petites et blanches ainsi qu'une peau blanche sont les plus froids et les plus secs comme les silures, les poissons tachetés de noirs [non identifiés] et ceux qui leur ressemblent.» (Harbī 2008: 256)

\section{ANIMAL TERRIEN}

Les animaux terriens ne peuvent pas quitter la terre vers l'air ou l'eau. Ils vivent sous terre, boivent rarement de l'eau et n'ont pas besoin de respirer l'air. Ils respirent la vapeur qui se forme dans leurs terriers, comme se forme la vapeur dans les puits, explique IbnAA. C'est cette même vapeur, dont la nature est proche de celle de l'eau, qui fait qu'ils n'ont pas besoin de boire et qui dote leur corps d'une certaine humidité. Parmi ces animaux, l'auteur cite les taupes (al- $\underline{h}$ uld), les gerboises (yarb $\bar{u})$, les hérissons (qunfud $)$ et les vipères (afä $\bar{\imath})$.

À la fin de son ouvrage et dans la cinquième section du chapitre III, IbnAA ne reprend pas ses exemples un à un comme il l'a fait avec les trois catégories précédentes, mais entame une discussion générale sur le mode de vie de ces animaux, les taupes et les serpents en particulier.

Nous voyons apparaître dans ce fragment du texte d'autres exemples d'animaux terriens qui n'ont pas été cités dans la première section $(b \bar{a} b)$ : les vers $(d \bar{u} d)$, les fourmis (naml), les scorpions ('aqrab). IbnAA explique que les animaux terriens sont de quatre types (Harbī 2008: 303):

- ceux qui creusent des galeries et vivent sous terre: ces derniers sont généralement dépourvus d'yeux et préferent ne pas sortir à la lumière comme les taupes (al-huld) et les lombrics ( $\underline{h}$ arātìnn); - ceux qui habitent sous terre mais chassent à sa surface comme les serpents (hayya) et les varans (waral);

- ceux qui habitent sous terre mais ne chassent pas et mangent les grains et les plantes comme les gerboises et les fouettequeue $(d a b b)$;

- ceux qu'il appelle hirršat al-ārd, petits animaux venimeux et «sales" (qadira) car générés dans la pourriture comme les scorpions ou les geckos ( $w a z a \dot{g})$.
Il est intéressant de noter que l'auteur n'utilise pas le terme hašáāat (Lamouchi Chebbi 2018), employé par Ğahiz ou Ihwān al-Ṣafāo pour désigner les insectes, reptiles et les petits mammifères (Lamouchi Chebbi \& Katouzian-Safadi 2019).

Les douze catégories d'animaux de nature composée sont les suivantes:

\section{FEU/Air}

Ces animaux volent haut dans l'air plus que dans la vapeur (au-dessus de l'eau). Ils sont de nature très chaude mais de complexion néanmoins plus humide que celle de l'animal de feu. Ils vivent et se reproduisent à la campagne et au bord de l'eau et se nourrissent de végétaux et de grains, tel est le cas par exemple des outardes (hubārā), des grues (karākī) ou des oies (iwazz).

\section{AIR/FEU}

De nature chaude et humide, les animaux de ce type volent plus souvent dans la vapeur que dans l'air. Ces oiseaux plutôt doux ont tendance à roucouler et à chanter. Ils vivent sur les arbres et la forme de leurs ailes fait qu' ils sont très rapides. Selon IbnAA, les colombes (al-warašān), les tourterelles (al-fawāhit) et les pigeons (al-hamām) en font partie.

\section{Feu/Eau}

Ce sont les rapaces d'eau de nature chaude et humide. Ils volent au-dessus de la vapeur et se nourrissent exclusivement de poissons vivants qu'ils pêchent à la surface de l'eau grâce à leurs longs becs. Une fois capturés et domestiqués, ces oiseaux sont capables de changer de régime et se nourrissent de petits animaux: il s'agit selon IbnAA du harìt et du nārmāhì, deux exemples d'oiseaux que nous n'avons pas pu identifier.

\section{EAU/FEU}

Leur composition en fait des oiseaux qui volent très haut et peuvent parcourir de longues distances. Cependant, à cause de l'abondance d'humidité dans leur complexion, ce sont des oiseaux lourds qui évitent l'effort quand ils cherchent la nourriture. Ils se nourrissent donc sur place, au bord de l'eau et mangent les poissons qu'ils trouvent dans les eaux peu profondes. Il n'a pas non plus été possible dans ce cas d'identifier les trois exemples d'oiseaux cités par IbnAA: al-bìdānì, al-nașrānī et al-wāmah.

\section{FEU/TERRE}

Ils sont de nature chaude et sèche; la dominance du feu dans leur composition fait que ces animaux volent haut dans l'air, au-dessus de la vapeur. Ils se nourrissent de cadavres et ne chassent pas. Ils habitent les montagnes et les falaises. Les corbeaux (al-girbān), les vautours (al-nisr) et les percnoptères (al-raham) sont parmi les exemples donnés par l'auteur pour cette catégorie.

\section{Terre/FeU}

Tout carnassier qui marche, court et saute fait partie de cette catégorie. Ces animaux sont agressifs et féroces à cause de l'élément feu, mais également courageux. Ils ne vivent 
pas en groupes et non seulement les mâles ne prennent pas soin de leurs petits mais ils peuvent aussi les dévorer, car ils ont le cœur dur, explique l'auteur. C'est le cas de la panthère (al-namir), du guépard (al-fahd), de la hyène (al-dabu') et d'autres encore.

\section{AIR/EAU}

Ce sont les oiseaux qui volent dans la vapeur juste au-dessus de l'eau. Ils nagent à la surface de l'eau et se nourrissent sur le rivage et dans les eaux peu profondes. Ils vivent et se reproduisent dans les bosquets au bord de l'eau comme dans le cas des bécassines des marais (al-šanaq).

\section{EAU/AIR}

Ces animaux sont aussi des oiseaux. Ils volent au-dessus des mers et font leurs nids dans les arbustes et dans les bois. Ils plongent dans l'eau pour se nourrir et peuvent rester longtemps en profondeur. IbnAA écrit qu'il a vu des poules d'eau (dağa $\check{g}$ al-máa), en grand nombre, nager à la surface de l'eau.

\section{AIR/TERRE}

L'air étant l'élément dominant dans leur constitution, ce sont encore une fois des oiseaux. Ils volent dans la vapeur et construisent leurs nids à la surface de la terre. IbnAA ne donne cependant pas d'exemple de ce type d'animaux.

\section{TERRE/AIr}

Ces animaux se nourrissent d'herbes, de roseaux, de brindilles et de graines. Leur mouvement est léger, à cause de l'élément air, et ils sont capables de sauter et de courir rapidement. Ils peuvent servir de nourriture aux humains et il est possible de domestiquer leurs petits étant donné que leur complexion est semblable à celle des animaux domestiques. Les antilopes $(a l-z \underline{i} \dot{i} \bar{a})$, les chevreuils (yahāminr) et les mouflons (arāwī) sont des exemples de ces animaux.

\section{EAU/TERre}

Il s'agit de tout animal qui vit dans l'eau et respire l'air mais peut aussi respirer l'eau. Ces animaux préfèrent l'eau à la terre et sont plus chauds que les poissons. IbnAA propose les exemples des grenouilles (dafädi'), des crabes (sarațānāt) et des tortues (salāhif). Il décrit même une expérience qui permet de vérifier qu'un crabe est capable de respirer sous l'eau.

\section{TERre/EAu}

Ces animaux naissent et vivent sur la terre mais peuvent se réfugier dans l'eau et y rester un certain temps. C'est le cas par exemple du pékan (dalaq) et de la loutre (kalbatu al-mā').

Après avoir exposé dans la première section du chapitre III ses seize catégories, l'auteur décrit dans la suite du texte (de la section 2 à la section 7 du chapitre III), avec plus de détails, la nature et les modes de vie des animaux déjà cités. Cette partie de l'ouvrage est malheureusement incomplète. Nous n'y trouvons que les quatre catégories simples et trois des catégories composées étudiées ci-dessus.
L'auteur n'avait peut-être pas pu terminer son œuvre pour une raison que nous ignorons. Quoi qu'il en soit, l'ensemble de cet ouvrage a été rassemblé au début du XIIIe siècle par 'Abd al-'Azīz Ibn 'Alī, un médecin expert comme il se décrit à la fin du texte. Ce dernier écrit qu'il a copié ce texte en trois ans.

Nous pensons que les deux derniers chapitres n'ont pas été écrits à la même période; en effet, les hiiršāat al-'ard (scorpions, fourmis, blattes et autres) qui n'apparaissaient pas dans les exemples du début du chapitre III (Harbī 2008: 261) trouvent ensuite leur place dans la catégorie simple des animaux terriens dans la section 5 (Harbī 2008: 302). Nous ne savons pas dans quelles conditions a été écrit cet ouvrage ni en combien de temps, mais nous pouvons remarquer que l'idée de la classification évolue tout au long du texte et s'enrichit en détails et en exemples.

Dans la première section du chapitre III, IbnAA prévoit une classification et y place les animaux selon l'espace qu'ils occupent; il établit ensuite des relations entre les qualités et le mode de vie, que résume la Figure 1, en mobilisant ses connaissances en médecine, en pharmacie, en géographie humaine, et en commençant sa classification par la déduction de la nature des qualités dominantes de l'objet observé (Fig. 1: D). Au chapitre I, IbnAA développe selon la zone géographique des traits de la physiognomonie humaine, tandis qu'au chapitre III, la même méthode est appliquée pour déterminer une physiognomonie appliquée aux animaux sauvages. L'auteur explique ainsi le mouvement, la rapidité ou la lenteur des gestes, les caractères moraux des animaux sauvages.

Contrairement à d'autres ouvrages arabes médiévaux concernés par la zoologie, cette œuvre ne comprend aucun aspect fantastique. Gāhiz par exemple, qui puise une grande partie de ses données dans des informations collectées oralement, laisse une place pour les animaux de légende. Son intérêt propre et la richesse des informations collectées permettent cela. IbnAA en revanche n'a pas de place dans cette structure pour les animaux légendaires. Plusieurs raisons peuvent être évoquées: il traite principalement des animaux connus; il serait malaisé de situer les animaux légendaires en rapport avec la supériorité de la raison humaine qui est le fondement de sa classification; enfin, les définir selon les quatre éléments dans un cadre lié strictement aux pratiques du monde médical serait difficile.

\section{CONCLUSION}

IbnAA se fonde sur les réflexions de ses prédécesseurs pour la classification des animaux sauvages. On peut remarquer des similitudes entre cette vision anthropocentrée du monde animal et la nôtre aujourd'hui ; mais nous ne pouvons nullement supposer que notre époque soit un aboutissement d'une pensée initiée au IXe siècle. Certes, son œuvre est citée par des savants postérieurs, mais ils n'ont pas tous suivi son chemin dans la classification des animaux sauvages. En effet, l'ensemble de la zoologie arabe n'a pas été radicalement modifiée par la pensée d'un seul auteur. Dans ce long cheminement de pensée qui sépare IbnAA de notre époque, les autres aspects des activités humaines, comme les progrès techniques et les outils inventés 
ont certainement eu des rôles importants et ont été des acteurs essentiels (Latour 2017). Cette discussion dépasse le cadre de notre travail; nos prétentions sont restreintes à l'analyse d'un court fragment d'histoire de la zoologie, histoire qui a poursuivi des chemins divers à travers des langues multiples dans des conditions culturelles variées.

Pour IbnAA, le noyau de la réflexion est constitué par la raison humaine, au sommet de son architecture classificatoire. Les animaux domestiques, grâce aux bénéfices (manāfí) qu'ils fournissent à l'homme, trouvent leur place dans cet édifice. Mais que faire, dans un tel univers des araignées, des serpents, des lions ? Comment expliquer philosophiquement l'existence des animaux sauvages? Ce débat a longtemps animé les cercles philosophiques, théologiques dans le monde médiéval, Arabe - Islamique ou latin. Al-Ğāhiz dans son Kitāb al Hayawān, distingue également les animaux domestiques $(a h l \bar{\imath})$ des sauvages (waḩšiz) et consacre de longues descriptions aux modes de vies des nuisibles (des moustiques ou des serpents) (Aarab et al. 2019: 64-74). Pour lui, ces animaux sont un témoignage de la grandeur du Créateur. (Aarab et al. 2000).

La méthodologie d'IbnAA pour la classification des animaux sauvages, peut se résumer ainsi :

- il se fonde sur la vérité absolue du monde sublunaire et corruptible au sens aristotélicien, c'est-à-dire les quatre éléments; - vérité qui est aussi indubitable que "les angles d'un triangle font deux angles droits", comme il le dit dans son ouvrage; - il collecte ensuite des observations bien réelles : lieu d'existence, mode de déplacement, qualité de la couverture du corps (peau, plumes), rapidité des mouvements, chasse, etc., et y associe également des observations provoquées (expérimentations); - à partir de ces observations, et avec les moyens à la disposition des naturalistes, des médecins et des pharmaciens (géographie humaine, physiognomonie, propriétés de la matière en pharmacopée...), il attribue une place à chaque animal sauvage, au sein de seize catégories, basées sur leur constitution élémentaire.

Des classifications semblables étaient déjà pratiquées par les pharmaciens de l'époque d'IbnAA.

Grâce cette classification, les animaux sauvages se trouvent inclus dans un "ensemble zoologique" dont les cases sont définies par la raison humaine et les observations et expérimentations agrémentent cet édifice. IbnAA trouve ainsi une solution rassurante et peu coûteuse en temps d'observation en les rendant accessibles à l'homme. Ces animaux indomptables, sauvages et incompris rentrent dans l'univers de la raison humaine et du compréhensible.

\section{Remerciements}

Nous remercions profondément le Professeur Ahmed Aarab pour les révisions de notre article. Nous remercions également nos relecteurs d'Antropozoologica pour leurs conseils judicieux. Pour l'identification des noms de certains animaux nous avons bénéficié de précieuses aides du Professeur Ahmed Aarab et de Philippe Provençal (pour les noms de certains poissons). Nos remerciements s'adressent également à M. le Professeur Roshdi Rashed qui a favorisé l'initiation de ces recherches dans l'équipe CHSPAM et au sein de l'équipe SPHERE du CNRS.

\section{BIBLIOGRAPHIE}

\section{SOURCES PRIMAIRES}

ARISTOTE: voir FILIUS 2018; Kruk 1979.

BASAJ A. Ḥ. (éd.) 2007. — Muhammad Ibn Mūsā al-Damìrì, Hayāt al-haywān al-kubrā. Dar al-Kutub al-ilmiya, Beirut, 1104 p.

Brague R. (trad.) 2003. - Räzì: la médecine spirituelle. Flammarion, Paris, $206 \mathrm{p}$.

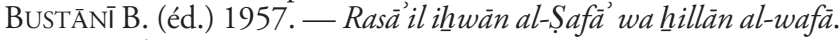
Dār Șāder, Beirut, 1968 p.

FILIUS L. S. 2018. - The Arabic Version of Aristotle's Historia Animalium, Book I-X of Kitāb al-Hayawān. Brill, London, xv + 539 p. (Coll. Aristoteles Semitico-Latinus; 23). https://doi. org/10.1163/9789004315969

Ğ̄ìIZ: voir HĀRūN 1996.

GALIEN : voir KÜHN 1821-1833; SEZGIN 2001.

Gamal A. S. (éd.) \& Dol M. W. (trad.) 1984. - Medieval Islamic Medicine: Ibn Ridwan's Treatise "On the Prevention of Bodily Ills in Egypt". University of California Press, Berkeley, 268 p. (Coll. Comparative Studies of Health Systems and Medical Care; 9)

Harbī A. A. (éd.) 2008.-Ibn Abì Al-Ash'ath, Al-Hayawān. Markaz al-Buhūth wa-al-Dirāsāt al-Islāmīyah, Bagdad, 325 p.

HĀrūN A. (éd.) 1996. — Al Ğăhiž, Kitāb al-Ḥayawān. Dār al-Ğīl, Beirut, 8 vol. (3830 p.).

HIPPOCRATE: voir JACQUES 2002.

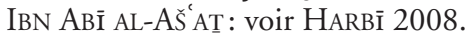

IBN ABī USSAYBÍA: voir SEZGIN 1995

IBN BUȚLĀN : voir SANAGUSTIN 2010.

IBN RIḌWĀN : voir GAMAL \& DOL 1984.

IHWW̄NN AL-ȘAFĀ' : voir BUSTĀNĪ 1957.

ISHĀQ IBN SULAYMĀN AL-ISRĀ'ĪLĪ: voir ȘABBAH 1992.

ISKANDAR A. Z. (éd.) 1961. — Rhazes' Kitāb al-muršid aw al-fușūl. Măgallat ma had al-maktutat al-arabiyya 1 (7).

ITANi A. (éd.) 1984. - Manafí al-adwiya wa daf madạrrihā. Dār Ihyä' al-'ulūm, Beirut, 296 p.

JouAnNA J. (éd.) 2002. - Hippocrate, La nature de l'homme. Deuxième édition anastatique augmentée et corrigée. Akademie Verlag, Berlin, $342 \mathrm{p}$.

Kraus P. (éd.) 1939. - Abi Bakr Mohammadi flii Zachariae Raghensis (Razis) Opera philosophica fragmentaque quae supersunt collegit et edidit Paulus Kraus. Pars prior. P. Barbey, Le Caire, iv +316 p.

KRUK R. 1979 - The Arabic Version of Aristotle's Parts of Animals. Book XI-XIV of the Kitāb al-Hayawān: a Critical Edition. Brill, Amsterdam, 256 p. (Coll. Aristoteles Semitico-Latinus; 2).

KÜHN K. G. (éd.) 1821-1833. - De simplicium medicamentorum temperamentis ac facultatibus libri XI, in Galeni opera omnia. Car. Cnoblochii, Leipzig, 20 Vol., 19000 p.

MoubaChir E.-A. (trad.) 1996. — Rãzī: Guide du médecin nomade. Actes Sud, Paris, 198 p.

QĀDİ BEN YŨSUF M. (éd.) 2006. — Al-Qazwīnì, 'Ağààib al-mahlūqāt wa garā' ib al-mawğūdāt. Maktabat al-țaqāfa al-dīniya, Le Caire, $405 \mathrm{p}$.

RĀZĪ : voir BRAGUE 2003; SIDDĪQī 1987; ITANI 1984; ISKANDAR 1961 ; Kraus 1939; Moubachir 1996.

ȘABAH M. (éd.) 1992. - Kitāb al-aǵdidya wa al-adwiya. Mua' ssasat 'Izz addīn liṭibā'a wā annašr, Beirut, 645 p.

SANAGUSTIN F. 2010. - Médecine et société en islam médiéval: Ibn Butlān ou la connaissance médicale au service de la communauté - Le cas de l'esclavage. Librairie orientaliste Paul Geuthner, Paris, 284 p.

SEZGIN F. (éd.) 1995. - Ibn abì Ușayba, 'Uyūn al-abnà fì țabaqāt al-atibbb$\vec{a}$. Institute for the history of Arabic-Islamic Science, Frankfurt am Main, 2 vols [1 re édition: Müller A. 1882-1884].

SEZGin F. (éd.) 2001. - Galen's Commentary on the Hippocratic

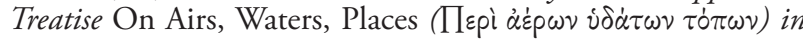
Arabic Translation. Institute for the History of Arabic-Islamic Science, Frankfurt am Main, 215 p. (Coll. Publications of the Institute for the History of Arabic-Islamic Science. Series C: Facsimilie Editions; 65). 
SidDīQī M. Z. (éd.)1928. — Firdows al-Hikma fì al-tibb. Mațba Āftāb, Berlin, 631 p.

SidDīQī H. (éd.) 1987. — Al-Manșūrì fì al-tịbb. Ma'had al-maḥtūțāt, Kuwait, 732 p.

ṬABARĨ : voir SIDDĪQĪ 1928.

\section{SOURCES SECONDAIRES}

AARAB A. 2001. - Étude analytique comparative de la zoologie médiévale, cas du Kitâb al-Haywân de al-Jâhiz. Thèse de $3 \mathrm{e}$ cycle, Faculté des Sciences Abdelmalek Essaädi, Tétouan, Maroc, 164 p.

AARAB A. 2017. — Predatory and anti-predatory strategies according to Ğāhiz through his work Kitāb al-Hayawān (The Book of Animals). Arabic Biology \& Medicine 5 (1): 1-19.

Aarab A. \& Lherminier P. 2015. — Le Livre des animaux d'AlJâhiz. L’Harmattan, Paris, 161 p.

AARAB A. \& PROVENÇAL P. 2013. - The orientation among birds according to Gähiz through his work Kitāb al-Hayawān (The Book of Animals). Arabic Biology and Medicine 1 (1): 25-38.

Aarab A. \& ProvençAL P. 2016. - La communication animale selon Ğāhiz, à travers son œuvre Kitāb al-Hayawān. Arabic Biology and Medicine 4 (1): 59-69.

Aarab A., Provencal P. \& Idaomar M. 2000. — Eco-Ethological data according to Jâhiz through his work Kitâb al-hayawân (The book of Animals). Arabica XLVII: 278-286.

Aarab A., Provencal P. \& IdaOmar M. 2003. —La méthodologie scientifique en matière zoologique de Jâhiz dans la rédaction de son œuvre Kitâb al-Hayawân. Anaquel de Estudios arabes 14: 5-19.

Aarab A., Lamouchi CHEbBi K. \& KatouZian-SAFadi M. 2019. The animal environment and human health: the approach followed by the medieval zoologist Ğähiz (ninth century), in Bretelle-Establet F., Gaille M. \& Katouzian-Safadi M. (éds), Making Sense of Health, Disease, and the Environment in Cross-Cultural History: the Arabic-Islamic World, China, Europe, and North America. Springer, Cham : 61-78.

Adamson P. 2012. - Abū Bakr al-Rāzì on Animals. Archiv für Geschichte der Philosophie 94 (3): 249-273.

Bosworth C. E., Van Donzel E., Heinrichs W. P. \& Pellat C. (éds) 1993. - Encyclopédie de l'Islam. Vol. 7, Mif-Naz. Brill, Leiden; Maisonneuve \& Larose, Paris.

BEN SAAD M. 2010. - La connaissance du monde vivant chez le savant al-Djâhiz (776-868). Thèse de doctorat en Épistémologie et en Histoire des Sciences, Université Paris 7 - Denis Diderot, Paris, 318 p.

BEN SAAD M. 2017. — Les poissons et les animaux aquatiques dans la classification des animaux d'al-Gâhiz (776-868). Arabic Biology and Medicine 5 (1): 29-72.

Ben SAAD M. \& KaTOUZIAN-SAFADi M. 2011. — Les Insectes dans la classification des animaux d'al-Djâhiz: entre mythe et raison, in TAlairach-Vielmas L. (éd.), Lost and Found: in Search of Extinct Species. Explora International Conference Proceedings, CAS/UTM, Toulouse, France: 228-250.

Ben Saad M., Katouzian-Safadi M. \& Provencal P. 2013. Réflexions sur un critère de classification des animaux chez alDjâhiz: le mode de reproduction chez les reptiles et les oiseaux, in Mlika H. (éd.), Approches en histoire des sciences arabes. Al-Mukhatabât (7): 69-86.

Boudon-Millot V. 2006. — Galien de Pergame face au mirage de la beauté parfaite. Bulletin de l'Association Guillaume Budé 1 (1): 127-141. https://doi.org/10.3406/bude.2006.2202

Bourgey L. 1955. - Observation et expérience chez Aristote. Vrin, Paris, $194 \mathrm{p}$.

Chebbi Lamouchi K. \& Katouzian-Safadi M. 2013. — L'étude des oiseaux dans Kitâb al-Hayawân de Jâhiz (776-868), in MuiKa H (éd.), Approches en histoire des sciences arabes. AlMukhatabât 7: 100-118. https://www.academia.edu/4471533/ AL-MUKHATABAT_JOURNAL_ISSUE_7_JULY_2013, dernière consultation: 16/07/2020.

DAHROUCH S. 2019. - Étude analytique et comparative de la nutrithérapie arabe clasique, cas de l'œuvre "Kitäb al-agdìya wa al-adwìya", écritpar Isaac Ben Solomon al-Isralìlì. Thèse de 3 e cycle, Université Abdelmalik Essaadi, Tanger, 287 p.

DAKHLIA J. 2002. - Les miroirs des princes islamiques : une modernité sourde. Annales HSS 5: 1191-1207.

DAVID R. 2004. - Rationality versus irrationality in Egyptian medicine in the pharaonic and graeco-roman periods, in HERMAN. F. J. (éd.), Magic and rationality in ancient Near Eastern and Graeco-roman medicine. Studies in Ancient Medicine 27 (15): 133-151.

DeBOTH Ricordel J. 2004. - "Al-Musta ini" les "Tables de médicaments simples" d'Ishak Ibn Biklârish. Édition, traduction française et analyse (des lettres alifà zay). Thèse de doctorat en Épistémologie et histoire des sciences, Université Paris 7 - Denis Diderot, Paris, 652 p.

Debru A. 1997. - Philosophie et pharmacologie: la dynamique des substances leptomères chez Galien, in Debru A. (éd.), Galen on pharmacology. Philosophy, History and Medicines. Brill, Leiden: 85-102.

Debru A. 2013. — Entre élément et tempérament: le corps phlegmatique. Medicina e storia 1 (2): 199-212.

DRUART T. A. 1996. — Al-Razi's conception of the soul: psychological background to this ethics. Medieval Philosophy \& Theology 5 (2): 245-263.

FouCHECOUR C.-H. DE 2010. — Le sage et le prince en Iran médiévalLes textes persans de morale et politique (IXe-XIIIe siècle). Paris, L'Harmatan, $511 \mathrm{p}$.

GuTAS D. 1998. - Greek thought, arabic culture. The Graeco-Arabic Translation Movement in Baghdad and Early 'Abbäsid Society (2nd4th/8th-10th centuries). Routledge, London, New York, 252 p. [Traduction française: Cheddadi A. 2005. - Pensée Grecque, culture arabe, le mouvement de traduction gréco-arabe à Bagdad et la société abbasside primitive (11e-IVe/VIIIe-Xe siècles). Aubier, Flammarion, Paris, 339 p.].

Hugonnard-Roche H. 1997. - Note sur Sergius de Resh'aina, traducteur du grec en syriaque et commentateur d'Aristote, in Endress G. \& KruK R. (éds), The Ancient Tradition in Christian and Islamic Hellenism. Studies on the Transmission of Greek Philosophy and sciences, dedicated to H. J. Drossaart. Lulofs, Leiden: 121-143.

JOLIVET J. 1971. — L'Intellect selon Kindi. Leiden, Brill, 166 p. (Coll. Publications de la Fondation De Goeje; XXII).

JOLIVET J. \& RASHED R. 1998. - Euvres philosophiques et scientifiques. Vol. 2, Métaphysique et cosmologie. Brill, Leiden, xiii + 243 p. (Coll. Islamic Philosophy and Science. Texts and Studies; 29).

JoUANNA J. 2013. - Greek Medicin from Hippocrates to Galen: selected papers. Brill, Leiden, 403 p. (Coll. Studies in Ancient Medicine; 40). https://doi.org/10.1163/9789004232549

KATOUZIAN-SAFADI M. 2004a. — La pharmacie arabe, in LECOURT D. (éd.), Dictionnaire de la pensée médicale. Presses Universitaires de France, Paris: 870-874.

KATOUZIAN-SAFADI M.2004b. — La cornue et l'alambic, instrument d'analyse et de preuve dans Les doutes sur Galien de Rāzīi, in MORELON R. \& Hasnawi A. (éds), De Zénon d'Elée à Poincaré: recueil d'études en hommage à Roshdi Rashed. Peeters, Louvain: 377-390.

KaTOUZIAN-SAFADI M. 2014. - Les pratiques médiévales aux frontières de l'alchimie et de la médecine. Ethnopharmacologia (52): 9-21.

KATOUZIAN-SAFADI M. \& BEN SAAD M. 2019. — Inhabited lands and temperaments. Observations and therapeutic solutions, the views of scientists and Medieval physicians: al-Ğāhiz (9th), Rāzī (9th-10th), Ibn Riḍwān (11th), in BRETELLE-EsTABLET F., Gaille M. \& Katouzian-Safadi M. (éds), Making Sense of Health, Disease, and the Environment in Cross-Cultural History. Springer, Cham: 209-238. (Coll. Boston Studies in the Philosophy and History of Science; 333).

KATOUZIAN-SAFADI M. \& KarAmati Y. 2018. — La tentation des médicaments composés chez Rāzī. Quelques rapprochements avec Ibn Sīnā et Ibn Rušd, in Rieder P. \& ZANeTTI Droz F. (éds), Materia medica. Savoirs et usages des médicaments aux époques médiévales et modernes. Droz, Paris: 21-52. 
KAZIMIRSKI B. 1860. - Dictionnaire Arabe-Français. Librairie du Liban, Beirut, 2 vol. (1392 + 1638 p.).

Kraus P. 1986. - Jābir Ibn Hayyān. Contribution à l'histoire des idées scientifiques dans l'islam, Jäbir et la science grecque. Les Belles Lettres, Paris, $406 \mathrm{p}$.

KRUK R. 1990. - A frothy bubble: spontaneous generation in the medieval Islamic tradition. Journal of Semitic Studies 37 (2): 265-282.

KRUK R. 2001. - Ibn abī L-Aš at Kitāb al-Hayawān: a scientific approach to anthropology, dietetics and zoological systematics. Zeitschrift für Geschichte der arabisch-islamischen Wissenschaften 14: 119-168.

KRUK R. 2005. - Suckling lamb and free range chicken: Ibn abi1-Ash ath on domestic animals and dietetics, in MARín M. \& De la Puente C. (éds), El banquete de las palabras:la alimentacion en los textos Arabes. Consejo superior de Investigaciones scientíficas, Madrid: 93-101. (Coll. Estudios Árabese Islámicos: Monografías; 10).

LAmOUCHI ChebBI K. 2018. - L'étude des insectes et autres petits animaux dans le Livre des animaux ou Kitāb al-Haywān de Gāhiz (776-968). Thèse de doctorat en Épistémologie et en Histoire des Sciences, Université Paris 7 - Denis Diderot, Paris, 242 p.

Lamouchi Chebbi K. \& Katouzian-Safadi M. 2019. - Métamorphoses et transformations subies par les insectes dans Kitāb al-Hayawān de Ğāhiz, in Förstel J. \& PlOUVIER M. (éds), L'animal: un objet d'étude. Éditions du Comité des Travaux historiques et scientifiques, Aubervilliers. https://books.openedition. org/cths/10143, dernière consultation le 19/06/2020.

LATOUR B. 2017. - Où atterrir? Comment s'orienter en politique. La Découverte, Paris, 160 p.

LE Coz R. 2004. — Les médecins nestoriens au Moyen Âge. L'Harmattan, Paris, $371 \mathrm{p}$

Manquat M. 1932. - Aristote naturaliste. Vrin, Paris, 128 p.

Pellegrin P. 1982. - La classification des animaux chez Aristote.
Statut de la biologie et unité de l'aristotélisme. Les Belles Lettres, Paris, $220 \mathrm{p}$.

Rashed M. 2000. - Abû Bakr al-Râzî et le Kalam. Mélanges de l'Institut dominicain d'études orientales du Caire 24: 39-54.

Rashed M. 2008. - Al-Farabī lost treatise On Changing Beings and the possibility of the eternity of the world. Arabic sciences and philosophy 18 (1): 19-58.

RICORDEL J. 2011. - Innovations en médecine arabe entre les IXe et XIIIe siècles. Revue d'Histoire de la Pharmacie 98 (370): 235-246.

SAAD A. 2002. - Les idées morales et métaphysiques chez Abu Bakr Ibn Zakariyy Al-Razi (Rhazes). Thèse de doctorat en Philologie arabe, École pratique des hautes Études, Paris, 562 p.

STEPHAN N. 1999. - Mathématique et pharmacologie dans l'euvre pharmaceutique du médecin-philosophe arabe al Kindî (ca. 801-866). Thèse de doctorat en Épistémologie et en Histoire des Sciences, Université Paris 7 - Denis Diderot, Paris, 531 p.

STEPHAN N. 2002. - Pionnier dans la recherche du degré de qualité des médicaments composés: le médecin et philosophe arabe alKindi (IXe siècle), in RAZAUllaH ANSARI S. M. (éd.), Science and Technology in the Islamic World. Brepols, Turnhout : 209-216.

STEPHAN N. 2011. — La théorie pharmacologique d'Ibn Rušd d'après le Kitāb al-Kulliyāt fì al-tibb, V. Principaux points de convergence et de divergence avec la théorie pharmacologique d'al-Kindī, in HasnaWI A. (eds), La lumière de l'intellect. La pensée scientifique et philosophique d'Averroès dans son temps. Peeters, Louvain: 147-155.

Ullmann M. 1995. — La médecine Islamique. Presses Universitaires de France, Paris, 159 p.

WilgauX J. 2008. - La physiognomonie antique: bref état des lieux, in DASEN V. \& WilgaUX J. (éds), Langages et métaphores du corps dans le monde antique. Presses Universitaires de Rennes, Rennes: 185-195.

ZuCKer A. 2005. - Aristote et les classifications zoologiques. Peeters, Louvain-La-Neuve, 368 p. 